\title{
EIRAS E BEIRAS: ATENÇÃO PSICOSSOCIAL A PESSOAS COM PROBLEMAS DECORRENTES DO USO DE DROGAS
}

\author{
EIRAS Y BEIRAS: ATENCIÓN PSICOSOCIAL A LAS PERSONAS CON \\ PROBLEMAS DERIVADOS DEL USO DE DROGAS \\ PLACES AND BORDERS: PSTCHOSOCIAL CARE FOR PEOPLE WITH \\ DRUG ABUSE
}

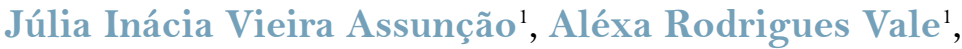 \\ Andrea Alves Oliveira ${ }^{1}$, Daniela Navarro Nilo ${ }^{1}$, \\ Doriane Santos Mariano ${ }^{1}$, Fernanda Gomes Palata ${ }^{1}$, \\ Marjorie Cristina Santana Fonseca ${ }^{1}$, Rafaela Ferreira Marques ${ }^{1}$, \\ Thaísa Borges Gomes ${ }^{1}$, Thamires Maria Miranda Santos ${ }^{1}$ e \\ Marcelo Dalla Vecchia ${ }^{1}$
}

${ }^{1}$ Universidade Federal de São Joao del-Rei, São Joao del-Rei/MG, Brasil

RESUMO: A oferta de estágios acadêmico-profissionalizantes é um recurso para reduzir lacunas relacionadas à formação para a atuação no campo de álcool e outras drogas. Apresentam-se reflexões acerca do projeto terapêutico singular (PTS) como tecnologia de cuidado na atenção psicossocial a pessoas com problemas decorrentes do uso de drogas e os seus efeitos da sua adoção na formação de estudantes de Psicologia. A abordagem da família como unidade de cuidado, o estabelecimento de vínculos e o respeito à autonomia foram ressaltados no decorrer do desenvolvimento da experiência de estágio. Nota-se que o ato de cuidar é central para a consolidação de um modelo de atenção contrahegemônico. Aponta-se ainda a necessidade de aprofundamento na construção teórica acerca do modelo de atenção de usuários de drogas na perspectiva da saúde pública.

PALAVRAS-CHAVE: Drogas (abuso); Redução de danos; Projeto terapêutico singular; Tratamento; Estágio.

RESUMEN: La oferta de prácticas académico-profesionalizantes es un recurso para reducir las ausencias relacionadas con la formación para la actuación en el campo de alcohol y otras drogas. Se presentan reflexiones acerca del proyecto terapéutico singular (PTS) como tecnología de atención en la atención psicosocial a personas con problemas derivados del uso de drogas y los efectos de su adopción en la formación de estudiantes de Psicología. El enfoque de la familia como unidad de cuidado, el establecimiento de vínculos y el respeto a la autonomía se resalta en el curso del desarrollo de la experiencia de práctica. Se observa que el acto de cuidar es central para la consolidación de un modelo de atención contra-hegemónico. Se apunta también la necesidad de profundizar en la construcción teórica acerca del modelo de atención de usuarios de drogas en la perspectiva de la salud pública.

PALABRAS CLAVE: Drogas (abuso); Reducción de daños; Proyecto terapéutico singular; Tratamiento; Práctica.

ABSTRACT: The provision of academic-professional internships is a resource to reduce training-related gaps in the field of alcohol and other drugs. Reflections on the singular therapeutic project (STP) are presented as a healthcare technology in psychosocial care for people with problems due to drug use, as well as its effects on the training of Psychology students. The approach of the family as a unit of care, the establishment of bonds and respect for autonomy were highlighted in the course of the internship experience. It is noted that the act of caring is central to the consolidation of a counter-hegemonic healthcare model. It is also pointed out the need to deepen the theoretical construction about the attention model of drug users from a public health perspective.

KEYWORDS: Drugs (abuse); Harm reduction; Singular therapeutic project; Treatment; Internship. 


\section{Introdução}

Os problemas relacionados ao uso de álcool e outras drogas têm relevância econômica, social e sanitária, fazendo-se necessária a mobilização de diversos setores das políticas públicas com vistas a manejar seus determinantes e consequências (Organização Mundial de Saúde [OMS], 2001). No Brasil, a emergência do consumo de álcool e outras drogas também tem demandado a elaboração e operacionalização de estratégias para equacionar a questão (Ministério da Saúde [MS], 2004),

Diante deste quadro, o MS formulou, no início do milênio, a Política para a Atenção Integral a Usuários de Álcool e Outras Drogas (PAIUAD), tendo como eixos norteadores a Lei 8.080/90, que institui o Sistema Único de Saúde (SUS), e a Lei 10.216/2001, marco legal da Reforma Psiquiátrica brasileira (MS, 2004). Contudo, é possível identificar um hiato histórico entre a elaboração de políticas específicas para os usuários de álcool e outras drogas e a PAIUAD. O Relatório Final da III Conferência de Saúde Mental ressaltou, dentre as conseqüências deste atraso, a persistente desassistência ou a assistência inadequada a essas pessoas (Conselho Nacional de Saúde [CNS], 2001).

As reduzidas oportunidades de formação inicial e continuada acerca das questões relacionadas ao uso de álcool e outras drogas também constituem impedimentos à condução adequada dos casos. Autores mencionam, ainda, dificuldades no acompanhamento longitudinal, e a reprodução tanto de estigmas e preconceitos acerca do uso de álcool e outras drogas quanto de concepções moralizantes acerca do usuário (Lima, Silva, Noto, Bonadio, \& Locatellli, 2015; Costa et al., 2015). Vale registrar que a formação de pessoal apto a atuar no SUS é preconizada na Lei n. 8.080/1990, como sendo responsabilidade das instituições formadoras e das políticas de saúde.

Segundo as Diretrizes Curriculares Nacionais da Graduação em Psicologia (DCN), a formação profissional em Psicologia deve contemplar conhecimentos que possibilitem o desenvolvimento de competências e habilidades voltadas para a atenção à saúde, tais como a temática álcool e outras drogas (Ministério de Educação, 2004). No entanto, para contemplar a pluralidade de dimensões que caracterizam o problema, são requeridas ações intersetoriais que transversalizem diversos campos de saber e práticas, como a assistência social, a segurança pública, a justiça, a educação, o trabalho, dentre outros (Passos \& Souza, 2011).

Neste sentido, o modelo de atenção à saúde dos usuários de drogas deve considerar a historicidade do sujeito e do seu contexto de vida, para que seja possível compreender e intervir diante do processo saúde-doença singular (Alves, 2009). As intervenções devem considerar os motivos subjacentes aos problemas relacionados ao uso para a redução das vulnerabilidades, promoção da saúde e prevenção dos riscos associados. Nota-se, no entanto, que ainda são predominantes iniciativas que adotam como única meta aceitável para o tratamento a abstinência, ignorando, assim, a singularidade da relação que cada indivíduo estabelece com as drogas (Machado \& Boarini, 2013; Pinho, Oliveira, \& Almeida, 2008).

A Redução de Danos (RD), neste sentido, é um "novo paradigma ético, clínico e político” (Passos \& Souza, 2011, p. 154) para as políticas de saúde, incluindo-se os cuidados direcionados aos usuários de drogas. Conforme estes autores definem, diferentemente do paradigma da abstinência, na RD não existe uma meta a priori como condição para o tratamento. Na RD, o próprio usuário é coparticipante e corresponsável pelo estabelecimento 
de suas metas terapêuticas junto dos técnicos e profissionais de saúde, visando um padrão de uso menos prejudicial.

Em síntese, entende-se que é fundamental delinear modos de lidar com a questão que considerem a singularidade característica das relações entre sujeito, droga e contexto. Não obstante, nas 'Referências Técnicas para a Atuação de Psicólogas/os em Políticas Públicas de Álcool e Outras Drogas', propostas pelo Conselho Federal de Psicologia (CFP), indica-se a relevância de se investir na discussão e aprofundamento da denominada 'clínica psicossocial' (CFP, 2013). Diante deste contexto, o presente trabalho propõe-se a apresentar reflexões que se originaram em um Programa de Extensão Universitária, que inclui uma proposta de estágio acadêmico-profissionalizante, acerca do cuidado a pessoas com problemas decorrentes do uso de álcool e outras drogas na perspectiva da atenção psicossocial.

\section{Método}

\section{Contexto da experiência}

O Programa de Extensão 'Eiras e Beiras: Atenção Psicossocial em Álcool e Outras Drogas - Consolidando Redes, Garantindo Direitos' foi desenvolvido no formato ora relatado durante os anos de 2014 e 2015. Dentre seus objetivos, estava a oferta de oportunidade formativa nessa área em cenários de prática diversos. Para tal, foram desenvolvidas atividades de atenção psicossocial em álcool e outras drogas, visando colaborar com a consolidação de uma rede de cuidados integrada e articulada, por meio de ações que priorizassem a garantia dos direitos humanos das pessoas atendidas. As dez primeiras autoras do presente trabalho foram as estagiárias componentes das atividades realizadas durante o ano de 2014, e o 11-autor é o coordenador do Programa de Extensão e supervisor acadêmico do projeto

As atividades ocorreram com a parceria de profissionais atuantes em equipes da Estratégia Saúde da Família (ESF) e do setor de gestão de pessoas da prefeitura municipal. Estas parcerias se firmaram a partir da demanda por colaborar no acolhimento de pessoas com problemas advindos do uso de álcool e outras drogas cadastradas por equipes da ESF, bem como pela atenção a servidores públicos municipais vivendo problemas nas relações de trabalho em decorrência do uso de álcool e outras drogas. A configuração do estágio consistiu em alocar três duplas de estagiárias em equipes da ESF de três bairros periféricos da cidade (uma dupla em cada área de abrangência); e duas duplas na Diretoria de Desenvolvimento de Pessoas (DDP) da prefeitura municipal.

\section{Procedimentos}

A operacionalização do estágio constituiu-se por meio da realização, pelas estagiárias, das seguintes atividades: (a) ambientação no território e na equipe: realização de visitas domiciliares aos casos identificados como foco de atenção e reconhecimento do processo de trabalho, no caso das duplas alocadas na DDP; (b) diagnóstico preliminar e continuado: amplo levantamento do caso, com histórico da condição, itinerário terapêutico, composição familiar etc; (c) formulação do Projeto Terapêutico Singular (PTS): definição de metas terapêuticas de curto, médio e longo prazos para abordagem dos casos e 
pactuação de compromissos com os atores mais comprometidos com a situação singular; (d) discussão e reavaliação periódica do PTS; e (e) confecção de diários de campo: relatos sobre os acontecimentos de cada ida a campo e as respectivas impressões subjetivas suscitadas em cada dupla de estagiárias.

O PTS foi a tecnologia de cuidado enfatizada nas atividades do estágio, constituindo-se em instrumento central para o seu desenvolvimento. A Portaria n. 3.088/2011, que regulamenta a RAPS, recomenda a adoção do PTS como instrumento fundamental de atenção psicossocial (MS, 2011). Boccardo, Zane, Rodrigues e Mângia (2011) o definem como uma tecnologia de cuidado organizada por meio de um conjunto de ações terapêuticas construídas coletivamente por uma equipe multidisciplinar. Por meio do PTS, busca-se o resgate da cidadania de pessoas em sofrimento mental, inclusive em decorrência de problemas devidos ao uso de álcool e outras drogas, secularmente estigmatizadas e vítimas de preconceitos, visando à conquista de sua autonomia e inserção social (Oliveira, 2013).

No que tange à noção de 'tecnologia de cuidado', Merhy (2014) sustenta que, por conta do objeto do trabalho em saúde não ser plenamente estruturado (o ser humano e seu sofrimento, a dor, o mal-estar, o desconforto etc), "suas tecnologias de ação mais estratégicas configuram-se em processos de intervenção em ato, operando como tecnologias de relações, de encontros de subjetividades” (p. 49). Estas tecnologias não-materiais são classificadas como 'tecnologias leves' que, segundo o autor, deveriam ser priorizadas no modelo tecnoassistencial de saúde contra-hegemônico. Entende-se, aqui, que o PTS é uma tecnologia de cuidado fundamental para mediar a operação de tecnologias leves junto de pessoas com problemas decorrentes do uso de álcool e outras drogas.

Como forma de ampliar o olhar para o contexto de vida das pessoas acompanhadas, recorreu-se ao genograma e ao ecomapa, instrumentos que possibilitam conhecer os aspectos estruturais e funcionais da família (Mello, Viera, Simpionato, Biasoli-Alves, \& Nascimento, 2005). O genograma possibilita compreender a composição e dinâmica familiar através da construção da "árvore da família” (p. 81), que caracteriza esta ferramenta (Mello et al., 2005). Quanto ao ecomapa, sua construção permite identificar as relações que a família estabelece com as outras pessoas e instituições presentes no ambiente no qual está inserida. Assim, segundo estes autores, torna-se mais fácil visualizar as estratégias de enfrentamento adotadas por cada família no decorrer de sua história, auxiliando na identificação de potencialidades e dificuldades para traçar o PTS.

\section{Resultados}

\section{A atenção psicossocial às pessoas com problemas decorrente do uso de drogas no 'Eiras e Beiras'}

O desenvolvimento do estágio acadêmico-profissionalizante possibilitou identificar três elementos como aspectos fundamentais da atenção psicossocial às pessoas com problemas decorrente do uso de drogas: (a) a abordagem familiar, (b) o estabelecimento de vínculos e (c) o respeito à autonomia. 
"A abordagem familiar". Constatou-se que as famílias das pessoas acompanhadas no estágio vivenciavam problemas de diversas ordens: conflitos entre os membros, histórico de ruptura de laços, conflito com a lei, violência intrafamiliar, dificuldades sócio-econômicas etc. Tais circunstâncias de potencial vulnerabilização eram frequentemente agravadas devido ao uso prejudicial de álcool e outras drogas por membro/s da família, notando-se, assim, a mútua influência entre a vulnerabilização da família e o consumo de substâncias psicoativas (Lima et al., 2018; Roehrs, Lenardt, \& Maftum, 2008).

A implementação de estratégias de produção de saúde centradas na família tem o potencial de enfatizar seu papel ativo no processo saúde-doença (Trad, 2010; Silva, Rodrigues, De Micheli, \& Andrade, 2015). Neste sentido, foi fundamental o uso do genograma e ecomapa, após treinamento para apropriação da técnica de utilização. Para a sua construção é necessário cultivar um vínculo entre o profissional de saúde e a família, de modo que esta sinta confiança em expor as suas particularidades (Mello et al., 2005).

O envolvimento da família tem um papel notável com relação ao uso de drogas entre seus membros (Pratta \& Santos, 2006; Roehrs, Lenardt, \& Maftum, 2008). Se, por um lado, o estabelecimento de vínculos de cooperação e solidariedade e a abertura a uma comunicação sem maiores barreiras entre os familiares desempenham papel protetivo, por outro, relações afetivas precárias, falta de apoio mútuo, dificuldade de comunicação e situações conflitivas permanentes constituem-se fatores de risco importantes para o estabelecimento de uma relação problemática com a droga (Paiva \& Ronzani, 2009).

Outro fator identificado nos casos acompanhados é a influência das práticas culturais familiares. Roehrs et al. (2008) apontam que a presença de drogas nos lares é comum e naturalizada, sendo raros os encontros familiares que não sejam permeados pelo consumo, quase sempre intenso, de bebidas alcoólicas e cigarro. Ademais, conforme os autores, o uso abusivo de psicofármacos é uma questão central no contex to histórico-social contemporâneo, ainda que na maioria das vezes seja negligenciada. Nos casos acompanhados, é possível perceber a 'familiaridade' com as substâncias psicoativas pelas pessoas acompanhadas pelo estágio desde a infância ou adolescência, pelo uso cotidiano no próprio domicílio.

"O estabelecimento de vínculos". Observou-se um significativo potencial terapêutico no decorrer do próprio processo de estabelecimento de vínculo entre as estagiárias e as pessoas atendidas e seus familiares. A criação de uma relação empática e compreensiva entre tais pessoas e as profissionais em formação contribuiu com o diagnóstico e o tratamento, bem como na adesão ao serviço e sucesso do tratamento (Caprara \& Rodrigues, 2004).

De igual modo, considera-se que a afetividade presente nos encontros entre as pessoas acompanhadas no estágio e as estagiárias teve papel importante no processo terapêutico. Era perceptível a melhora, por parte das pessoas acompanhadas, no autocuidado, no cuidado com o domicílio e no cuidado com os demais em seu entorno. Frequentemente, eram dirigidas a elas perguntas sobre alimentação saudável e a respeito dos medicamentos em uso para condições crônicas associadas ou não ao consumo de álcool e outras drogas.

Esta vivência permitiu às estagiárias elaborarem suas dúvidas em relação ao medo de estarem desempenhando uma prática tutelar, uma vez que as atividades do estágio envolviam o acompanhamento dos sujeitos em diversos espaços cotidianos (Borges, Omoré, Krenkel, \& Schneider, 2017). Percebeu-se, pelo contrário, que este movimento colaborou, paulatinamente, com a produção de autonomia e protagonismo dos sujeitos acompanhados. 
Considerando-se que o saber técnico-científico adquirido na formação do profissional de saúde deve estar articulado às especificidades culturais e socioeconômicas de cada comunidade, as estudantes puderam identificar que o acompanhamento aos sujeitos em seus contextos de vida possibilitava intervenções terapêuticas mais qualificadas. Para tanto, é preciso que os diversos saberes sejam igualmente considerados, superando-se as relações de hierarquia características do modelo biomédico (Caprara \& Rodrigues, 2004).

“O respeito à autonomia”. A premissa ético-política de que as práticas de cuidado devem ter um caráter emancipatório (Caprara \& Rodrigues, 2004) pautou a atuação das estagiárias, que eram orientadas a manter uma escuta atenta e respeitosa, problematizando junto das pessoas acompanhadas os riscos e danos a que poderiam estar expostas devido ao uso de drogas. Evitando uma postura intrusiva e moralista, os diálogos travados buscaram a promoção de empoderamento de sujeitos para os quais as substâncias psicoativas ocupavam um lugar central na vida, gerando prejuízos nas relações com a família, trabalho, lazer etc.

O respeito ao desejo do sujeito em modificar ou não seu padrão de uso, fazendo valer a sua liberdade de escolha, constitui-se pressuposto fundamental da RD, enfocando, portanto, o direito de todos à saúde, respeitando-se a liberdade individual daquele que não quer, não consegue ou não pode se manter abstinente (Machado \& Boarini, 2013). Nesta direção, era perceptível que, embora os casos acompanhados tivessem pontos em comum, a condução terapêutica pelas estagiárias diferiu consideravelmente. As estratégias adotadas para a operacionalização do PTS devem ter como foco o sujeito, que constitui a sua subjetividade em um contex to sócio-histórico-cultural específico (Oliveira, 2013).

A concepção de 'sucesso do tratamento' também foi problematizada pelas estagiárias. No início do estágio, as estudantes pontuaram o estranhamento ao entrar em contato com sujeitos cujo estilo de vida era percebido como muito divergente do seu. No decorrer das atividades, porém, passaram a notar que abster-se de posições morais e juízos de valor na atuação surtia efeito positivo no vínculo, na adesão à proposta terapêutica e no próprio tratamento como um todo. Isso fomentou uma relação mais honesta entre as partes. Com isso, o trabalho de revisão das metas traçadas no PTS, quando necessário, tor nava-se facilitado.

Inicialmente, quando os sujeitos acompanhados relatavam episódios de uso abusivo de álcool e outras drogas, estes se esquivavam das estagiárias, e eram perceptíveis sentimentos de vergonha e fracasso pelos 'lapsos' ou 'recaídas', demonstrando sua apropriação do vocabulário típico do paradigma hegemônico da abstinência. Mediante o esclarecimento de que a proposta do estágio não pressupunha necessariamente a abstinência como condição para o tratamento, estabeleceram-se relações mais abertas. 'Lapsos' e 'recaídas' foram aceitos tanto pelos sujeitos quanto pelas estagiárias como parte do tratamento. Se antes as 'recaídas' eram concebidas como a neutralização dos esforços até então realizados, o contato posterior passou a ser considerado como uma oportunidade de continuidade do tratamento. Assim, as 'recaídas' sugeriam a necessidade de reavaliação das metas do PTS.

Estas táticas divergem das estratégias repressivas adotadas pelas políticas proibicionistas baseadas no medo da repressão, na intimidação moralista e na imposição da abstinência (Machado \& Boarini, 2013). Além da ineficácia destas condutas, historicamente hegemônicas, deve-se salientar que se trata de posições eticamente questionáveis no que tange à atuação profissional do psicólogo (CFP, 2013). 


\section{Considerações finais}

Ao apontar alguns eixos norteadores (abordagem familiar, estabelecimento de vínculos e respeito à autonomia) para o estabelecimento dos fundamentos da clínica no âmbito da atenção psicossocial em álcool e outras drogas, entende-se que a adoção do PTS como tecnologia de cuidado, mediando as tecnologias leves, é fundamental. Entretanto, tal como se pôde observar nas atividades do estágio em análise, o PTS constitui-se uma importante estratégia a ser considerada, e não uma prescrição engessada aos trabalhadores de saúde como mais um 'protocolo', o que poderia redundar em uma indesejável burocratização.

As relações de confiança e colaboração construídas entre as estagiárias do Programa de Extensão e os usuários, e entre elas e a equipe de trabalho dos serviços, foram benéficas para a adesão do usuário à proposta de tratamento. Assim, a coprodução e a cogestão dos PTS foi viabilizada tendo em vista a implicação dos diversos atores envolvidos nos casos. Nessa direção, as teorias e técnicas envolvidas na formação do psicólogo podem contribuir para o trabalho de equipes envolvidas com a atenção psicossocial de pessoas com problemas decorrentes do uso de álcool e outras drogas. Além disso, a inclusão de estagiárias/os pode fomentar a problematização das práticas vigentes, contribuindo com a formulação de estratégias de cuidado consoantes com a perspectiva ético-política da RD.

Os psicólogos têm sido demandados a atuar em espaços diversos daqueles de atuação hegemônica, caracterizada pelo atendimento clínico individual em consultório. Diante de realidades socioculturais bastante diversificadas, a simples transposição deste modo de atuação é tão incoerente quanto ineficaz. Desse modo, faz-se imperiosa a reformulação dos projetos político-pedagógicos dos cursos de formação profissional no sentido de contemplar nos currículos uma atuação que enfatize o território (CFP, 2013).

O campo de atuação é desafiado constantemente com novas demandas, tendo em vista a multideterminação e complexidade da temática do uso, prejudicial ou não, de álcool e outras drogas. Assim, acadêmicos e profissionais na linha de frente de atuação devem assumir o compromisso de uma interação dialógica constante, buscando sempre a consolidação de condutas éticas para a defesa dos direitos humanos e o resgate da cidadania de indivíduos historicamente vítimas de estigma, preconceito e discriminação social. 


\section{Referências}

Alves, V. S. (2009). Modelos de atenção à saúde de usuários de álcool e outras drogas: discursos políticos, saberes e práticas. Cadernos de Saúde Pública, 25(11), 2309-2319. doi: 10.1590/ S0102-311X2009001100002

Boccardo, A. C. S., Zane, F. C., Rodrigues, S., \& Mângia, E. F. (2011). O projeto terapêutico singular como estratégia de organização do cuidado nos serviços de saúde mental. Revista de Terapia Ocupacional (USP), 22(1), 85-92. doi: 10.11606/issn.2238-6149.v22i1p85-92

Borges, C. D., Omoré, C. L. O. O., Krenkel, S., \& Schneider, D. R. (2017). Família, redes sociais e o uso de drogas: tensionamento entre o risco e a proteção. Pesquisas e Práticas Psicossociais, 12(2), 405-421. http://pepsic.bvsalud.org/pdf/ppp/v12n2/12.pdf

Caprara, A. \& Rodrigues, J. (2004). A relação assimétrica médico-paciente: repensando o vínculo terapêutico. Ciência \& Saúde Coletiva, 9(1), 139-146. doi:10.1590/S141381232004000100014

Conselho Nacional de Saúde [CNS]. (2001). III Conferencia Nacional de Saúde Mental: Caderno Informativo. Brasília, DF: Ministério da Saúde.

Conselho Federal de Psicologia [CFP]. (2013). Referências Técnicas para a Atuação de Psicólogas/os em Políticas Públicas de Álcool e Outras Drogas. Brasília, DF: Autor.

Costa, P. H. A., Mota, D. C. B., Cruvinel, E., Paiva, F. S., Gomide, H. P., Souza, I. C. W., Martins, L. F., Sillveira, P. S., \& Ronzani, T. M. (2015). Capacitação em álcool e outras drogas para profissionais da saúde e assistência social: relato de experiência. Interface - Comunicação, Saúde, Educação, 19(53), 395-404. doi: 10.1590/1807-57622014.0607

Lei $n$. 8.080, de 19 de setembro de 1990. (1990). Dispõe sobre as condições para a promoção, proteção e recuperação da saúde, a organização e o funcionamento dos serviços correspondentes e dá outras providências. Brasília, DF: Presidência da República.

Lei n. 10.216, de 06 de abrill de 2001. (2001). Dispõe sobre a proteção e os direitos das pessoas portadoras de transtornos mentais e redireciona o modelo assistencial em saúde mental. Brasília, DF: Presidência da República.

Lima, D. W. C., Leite, A. C. Q. B., Vieira, A. N., Leite, A. R., Luis, M. A. V., Azevedlo, L. D. S., \& de Melo, J. A. L. (2018). Necessidades de saúde de familiares de usuários de substâncias psicoativas. Revista Eletrônica de Enfermagem, 20(12), 1-10. doi: 10.5216/ree.v20.47410

Lima, J. M., Bonadio, A., Sillva, E. A.; Noto, A. R.; Locatelli, D. P. (2015). A educação permanente em álcool e outras drogas: marcos conceituais, desafios e possibilidades. In T. M. Ronzani, P. H. A. C. Costa, D. C. B. Mota, T. J. Laport (Orgs.), Redes de atenção aos usuários de drogas: políticas e práticas (pp. 115-187). São Paulo: Cortez.

Machado, L. V. \& Boarini, M. L. (2013). Políticas sobre drogas no Brasil: a estratégia de redução de danos. Psicologia: Ciência e Profissão, 33(3), 580-595. doi: 10.1590/S141498932013000300006

Mello, D. F., Viera, C. S., Simpionato, E., Biasoli-Alves, Z. M. M., \& Nascimento, L. C. (2005). Genograma e ecomapa: possibilidades de utilização na Estratégia de Saúde da Família. Revista Brasileira de Crescimento e Desenvolvimento Humano, 15(1), 79-89.

Merhy, E. E. (2014). Saúde: a cartografia do trabalho vivo (4a ed.). São Paulo: Hucitec.

Ministério da Ed ucação.(2004). ParecerCNE/CES n. 62, 19 de fevereiro de 2004. Diretrizes Curriculares Nacionais para os cursos de graduação em Psicologia. Brasília, DF: Autor. Recuperado de http://portal.mec.gov.br/index.php?option=com_docman\&view=download\&alias=7690rces004-08-pdf\&category_slug=marco-2011-pdf\&Itemid=30192

Ministério da Saúde. (2004). A Política do Ministério da Saúde para a Atenção Integral a Usuários de Álcool e outras Drogas. Brasília, DF: Autor. 
Oliveira, G. N. (2013). O projeto terapêutico singular. In G. W. S. Campos \& A. V. P. Guerrero (Orgs.), Manual de práticas de atenção básica: saúde ampliada e compartilhada (3a ed., pp. 283-297). São Paulo: Hucitec.

Organização Mundial de Saúde. (2001). Relatório sobre a Saúde no Mundo 2001 - Saúde Mental: Nova Concepção, Nova Esperança. Genebra: Autor.

Paiva, F. S. \& Ronzani, T. M. (2009). Estilos parentais e consumo de drogas entre adolescentes: revisão sistemática. Psicologia em Estudo, 14(1), 177-183. doi: 10.1590/S141373722009000100021

Passos, E. H. \& Souza, T. P. (2011, janeiro/abril). Redução de danos e saúde pública: construções alternativas à política global de "guerra às drogas". Psicologia \& Sociedade, 23(1), 154-162. doi: 10.1590/S0102-71822011000100017

Pinho, P. H., Oliveira, M. A., \& Almeida, M. M. (2008). A reabilitação psicossocial na atenção aos transtornos associados ao consumo de álcool e outras drogas: uma estratégia possível? Revista de Psiquiatria Clínica, 35(1), 82-88. doi: 10.1590/S0101-60832008000700017

Portaria n 3.088/MS/GM, de 23 de dezembro de 2011. (2011). Institui a Rede de Atenção Psicossocial para pessoas com sofrimento ou transtorno mental e com necessidades decorrentes do uso de crack, álcool e outras drogas, no âmbito do Sistema Único de Saúde. Brasília, DF: Ministério da Saúde.

Pratta, E. M. M. \& Santos, M. A. (2006). Reflexões sobre as relações entre drogadição, adolescência e família: um estudo bibliográfico. Estudos de Psicologia, 11(3), 315-322. doi: 10.1590/S1413-294X2006000300009

Roehrs, H., Lenardit, M. H., \& Maftum, M. A. (2008). Práticas culturais familiares e o uso de drogas psicoativas pelos adolescentes: reflexão teórica. Escola Anna Nery Revista de Enfermagem, 12(2), 353-357. Recuperado de http://www.scielo.br/pdf/ean/v12n2/v12n2a24.pdf

Silva, E. A., Rodrigues, T. P., Micheli, D., \& Andrade, A. L. M. (2015). Estratégias utilizadas no tratamento de famílias com usuários de substâncias. Revista Psicologia em Pesquisa, 9(2), 198-204. doi: 10.5327/Z1982-1247201500020010

Trad, S. (2010). Princípios e desafios para a integração da família nas políticas de drogas. In L. A. B. Trad (Org.), Família contemporânea e saúde: significados, práticas e políticas públicas (pp. 179-197). Rio de Janeiro: Fiocruz. 


\title{
JÚLIA INÁCIA VIEIRA ASSUNÇÃO
}

http://orcid.org/OOOO-0001-6407-1666

Especialista em Psicologia Hospitalar. Associação de Assistência à Criança e ao Adolescente Cardíacos e aos Transplantados do Coração (ACTC) de São Paulo/SP.

Endereço para correspondência: Rua Bahia, 153, Centro, Ritápolis/MG, Brasil. CEP 36.335-OOO

E-mail: assuncaojulia@hotmail.com

\section{ALÉXA RODRIGUES VALE}

http://orcid.org/OOOO-0003-2855-2264

Mestre em Psicologia. Centro de Referência Especializado para População em Situação de Rua de Belo Horizonte, MG.

E-mail: alexafdj@gmail.com

\author{
ANDREA ALVES OLIVEIRA \\ http://orcid.org/OOOO-0002-3364-3569 \\ Especialista em Gestão da Organização Pública de Saúde. Departamento de Psicologia, \\ Centro Universitário UNIFACIG, MG. \\ E-mail: andrea.alvesoliveira@yahoo.com.br
}

\section{DANIELA NAVARRO PAIVA NILO}

http:/ / orcid.org/OOOO-0002-5213-038X

Psicóloga, Residente em Psicologia do Programa de Residência Integrada Multiprofissional em Atenção Hospitalar do HU-UFJF, MG.

E-mail: dani_npn@hotmail.com

\section{DORIANE SANTOS MARIANO}

http://orcid.org/OOOO-0OO1-5772-8682

Psicóloga, Associação de Pais e Amigos dos Excepcionais de São João del-Rei, MG.

E-mail: dori_anes@hotmail.com

\section{FERNANDA GOMES PALATA}

http://orcid.org/OOOO-0002-9695-3898

Mestre em Psicologia Forense, Universidade Lusófona de Humanidades e Tecnologia, Lisboa, Portugal.

E-mail: fernandalaura1000@hotmail.com

\section{MARJORIE CRISTINA SANTANA FONSECA}

http:/ / orcid.org/OOOO-OOO1-5788-4271

Psicóloga, Setor Educativo do Museu de Congonhas/MG.

E-mail: marjorie-cris@hotmail.com 


\title{
RAFAELA FERREIRA MARQUES
}

http:/ / orcid.org/0000-0002-6507-0734

Psicóloga, residente do Programa Multiprofissional em Saúde, Faculdade de Medicina,

Universidade Federal de Uberlândia, MG.

E-mail: psirafamarques@gmail.com

\section{THAIISA BORGES GOMES}

http:/ / orcid.org/OOOO-0003-2513-7807

Mestre em Psicologia, atua em consultório particular.

E-mail: thabog@gmail.com

\author{
THAMIRES MARIA MIRANDA SANTOS \\ http:/ / orcid.org/O000-0003-3079-2 144 \\ Psicóloga. Prefeitura de Santo Antônio do Amparo / MG. \\ E-mail: thamires_mms@hotmail.com
}

\section{MARCELO DALLA VECCHIA}

http://orcid.org/OOOO-0001-7537-3598

Doutor em Saúde Coletiva. Departamento de Psicologia, Universidade Federal de São

João del-Rei, MG.

E-mail: mdvecchia@ufsj.edu.br

\begin{tabular}{|c|c|}
\hline Histórico & $\begin{array}{l}\text { Submissão: } 18 / 04 / 2017 \\
\text { Revisão: } 17 / 11 / 2018 \\
\text { Aceite: } 11 / 12 / 2018\end{array}$ \\
\hline Contribuição dos autores & $\begin{array}{l}\text { Concepção: JIVA, RFM, MDV } \\
\text { Coleta de dados: TBG, TMMS, RFM, AAO, MCSF, FGP, } \\
\text { ARV, DSM, DNN, JIVA } \\
\text { Análise de dados: MDV, TBG, TMMS, RFM, AAO, } \\
\text { MCSF, FGP, ARV, DSM, DNN, JIVA } \\
\text { Elaboração do manuscrito: MDV, TBG, TMMS, RFM, } \\
\text { AAO, MCSF, FGP, ARV, DSM, DNN, JIVA } \\
\text { Crítico revisões de conteúdo intelectual importante: } \\
\text { JIVA, MDV } \\
\text { Aprovação final do manuscrito: MDV, TBG, TMMS, } \\
\text { RFM, AAO, MCSF, FGP, ARV, DSM, DNN, JIVA }\end{array}$ \\
\hline Financiamento & $\begin{array}{l}\text { Programa de Bolsas de Extensão, Pró-Reitoria de Ex- } \\
\text { tensão e Assuntos Comunitários, Universidade Federal } \\
\text { de São João del-Rei - PIBEX/PROEX/UFSJ (Edital nº } \\
\text { 014/2013/PROEX). }\end{array}$ \\
\hline $\begin{array}{l}\text { Consentimento de uso de } \\
\text { imagem }\end{array}$ & Não se aplica \\
\hline $\begin{array}{l}\text { Aprovação, ética e } \\
\text { consentimento }\end{array}$ & Não se aplica \\
\hline
\end{tabular}

